\title{
miRNA-10a promotes cancer cell proliferation in oral squamous cell carcinoma by upregulating GLUT1 and promoting glucose metabolism
}

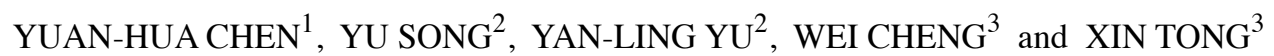 \\ ${ }^{1}$ Department of Prosthodontics, Nanjing Stomatological Hospital, Medical School of Nanjing University, Nanjing, \\ Jiangsu 210008; ${ }^{2}$ Department of Orthodontics, Qingdao Stomatological Hospital, Qingdao, Shandong 266001; \\ ${ }^{3}$ Department of Dental Implantation, Nanjing Stomatological Hospital, Medical School of \\ Nanjing University, Nanjing, Jiangsu 210008, P.R. China
}

Received September 20, 2018; Accepted March 22, 2019

DOI: $10.3892 / \mathrm{ol} .2019 .10257$

\begin{abstract}
MicroRNA-10a (miRNA-10a) promotes lung cancer; however, to the best of our knowledge, its involvement in other cancer types is unknown. The present study aimed to investigate the role of miRNA-10a in oral cancer. Expression levels of miRNA-10a and glucose transporter 1 (GLUT1) in tumor tissues and adjacent healthy tissues obtained from patients with oral squamous cell carcinoma (OSCC) were detected by reverse transcription-quantitative polymerase chain reaction. Correlation analysis between the expression levels of miRNA-10a and GLUT1 was performed using Pearson's correlation coefficient. It was identified that miRNA-10a and GLUT1 were upregulated in tumor tissues compared with adjacent healthy tissues of patients with OSCC. Expression levels of miRNA-10a and GLUT1 were positively correlated in tumor tissues but not in adjacent healthy tissues. In addition, miRNA-10a overexpression promoted glucose uptake and upregulated GLUT1 in OSCC cells. Furthermore, GLUT1 overexpression promoted glucose uptake; however, no significant increase in the expression level of miRNA-10a in OSCC cells was detected. Overexpression of miRNA-10a and GLUT1 promoted OSCC cell proliferation, while GLUT1-knockdown inhibited OSCC cell proliferation. GLUT1-knockdown also attenuated the enhancing effect of miRNA-10a overexpression on cancer cell proliferation. Therefore, miRNA-10a may promote cancer cell proliferation in OSCC by upregulating GLUT1 and promoting glucose metabolism.
\end{abstract}

Correspondence to: Dr Wei Cheng, Department of Dental Implantation, Nanjing Stomatological Hospital, Medical School of Nanjing University, 30 Zhongyan Road, Nanjing, Jiangsu 210008, P.R. China

E-mail: iptph58@163.com

Key words: oral squamous cell carcinoma, microRNA-10a, glucose transporter 1, glucose uptake, proliferation

\section{Introduction}

As a type of malignancy that develops in the hard palate, lips, the anterior two-thirds of the tongue, the upper and lower alveolar ridges, buccal mucosa, retromolar trigone, sublingual region and floor of the mouth, oral cancer affected $>0.0001 \%$ of people between 2000 and 2010 in the USA $(1,2)$. The incidence rate of oral cancer has exhibited an increasing trend in recent years (3). The occurrence of oral cancer has been demonstrated to be significantly associated with smoking, alcohol drinking, poor diet, poor oral hygiene and human papilloma virus infection (4). However, to the best of our knowledge, the molecular mechanism of the pathogenesis of oral cancer remains unknown (5), which hinders the development of effective treatment strategies.

Tumor cells are characterized by abnormally accelerated energy metabolism (6). Therefore, inhibition of energy metabolism is considered a promising treatment target for cancer therapy (7). Glucose transporter 1 (GLUT1), also termed facilitated glucose transporter member 1, is a uniporter protein that facilitates the transport of glucose to mammalian cells (8). A number of studies have reported that GLUT1 is abnormally upregulated in human cancer and promotes cancer development and progression by regulating cancer cell glucose metabolism $(9,10)$. MicroRNAs (miRNAs) are critical factors in cancer biology (11). MicroRNAs in certain cases participate in cancer biology by affecting energy metabolism (12), particularly by regulating the expression of GLUT1 (13). miRNA-10a has been characterized as an oncogenic miRNA in lung cancer (14). In lung cancer, miRNA-10a is upregulated and the overexpression of miRNA-10a promotes cancer development and progression via interactions with phosphatase and tensin homolog signaling (14). However, to the best of our knowledge, no study has investigated the involvement of miRNA-10a in energy metabolism or the regulation of GLUT1 expression. The present study revealed that miRNA-10a may promote cancer cell proliferation in oral squamous cell carcinoma (OSCC), a major type of oral cancer, by serving as an upstream activator of GLUT1 and promoting glucose metabolism. 


\section{Materials and methods}

Human materials and cell lines. Tumor tissue and adjacent healthy tissue samples were obtained from 52 patients with OSCC who were treated at Nanjing Stomatological Hospital (Nanjing, China) between July 2014 and July 2018. The inclusion criteria were as follows: i) Patients were diagnosed with OSCC by pathological biopsies; ii) patients with complete medical records; iii) patients with no history of another type of malignancy; and iv) patients provided written informed consent. The exclusion criteria were as follows: i) Patients who had been diagnosed with multiple diseases; and ii) patients who had received treatment prior to admission at Nanjing Stomatological Hospital. In total, the present study included 29 males and 23 females, with an age range of 33-65 years and a mean age of $45.3 \pm 4.4$ years. The current study was approved by the Ethics Committee of Nanjing Stomatological Hospital (Nanjing, China).

The OSCC cell lines SCC090 and SCC25 were purchased from American Type Culture Collection (ATCC; Manassas, VA, USA). Cells were cultured in Eagle's Minimum Essential Medium (ATCC) containing $2 \mathrm{mM}$ L-glutamine (Sangon Biotech Co., Ltd., Shanghai, China) and $10 \%$ fetal bovine serum (FBS, Sangon Biotech Co., Ltd.) at $37^{\circ} \mathrm{C}$ with $5 \% \mathrm{CO}_{2}$.

RNA extraction and reverse transcription-quantitative polymerase chain reaction (RT-qPCR). GenElute ${ }^{\mathrm{TM}}$ Total RNA Purification kit (Sigma-Aldrich; Merck KGaA, Darmstadt, Germany) was used to extract total RNA from tumor tissues, adjacent healthy tissues and in vitro cultured cells. For miRNA extraction, TaqMan miRNA Isolation kit (Applied Biosystems; Thermo Fisher Scientific, Inc., Waltham, MA, USA) was used. RevertAid RT Reverse Transcription kit for total RNA reverse transcription (Thermo Fisher Scientific, Inc.) and TaqMan microRNA Reverse Transcription kit (Applied Biosystems; Thermo Fisher Scientific, Inc.) was used for miRNA reverse transcription. qPCR was performed using a Luna ${ }^{\circledR}$ Universal One-Step RT-qPCR kit (catalog no. E3005; New England BioLabs, Inc., Ipswich, MA, USA) or miScript SYBR Green PCR kit (Qiagen GmbH, Hilden, Germany), according to the manufacturers' protocols. Primers for miRNA-10a, GLUT1 and the endogenous control U6 were designed and synthesized by Sangon Biotech Co., Ltd. The following primer sequences were used: miRNA-10a forward, 5'-GGAGGGGTACCAGAATCC CATTTTGGCCA-3' and reverse, 5'-GGAGGAAGCTTGCGG AGTGTTTATGTCAACT-3'; GLUT1 forward, 5'-CATCCT TATTGCCCAGGTGTTT-3' and reverse, 5'-GAAGACGAC ACTGAGCAGCAGA-3'; and U6 forward, 5'-GCTTCGGCA GCACATATACTAAAAT-3' and reverse, 5'-CGCTTCACG AATTTGCGTGTCAT-3'. All PCR reactions were performed on a StepOnePlus real-time PCR system (Applied Biosystems; Thermo Fisher Scientific, Inc.) with the following conditions: $95^{\circ} \mathrm{C}$ for $55 \mathrm{sec}, 40$ cycles at $95^{\circ} \mathrm{C}$ for $18 \mathrm{sec}$ and $60.5^{\circ} \mathrm{C}$ for $35 \mathrm{sec}$. Ct values were normalized using $2^{-\Delta \Delta \mathrm{Ca}}$ method (15).

Cell transfection. MISSION ${ }^{\circledR}$ microRNA Mimic hsa-miR-10a (3'-CAAAUUCGUAUCUAGGGGAAUA-5') and scrambled negative control miRNA (cat. no., SIC001-1NMOL) were purchased from Sigma-Aldrich; Merck KGaA. Vectors expressing GLUT1, GLUT1 small interferring RNA (siRNA) (5'-CCUCUUUGUUAAUCGCUUU-3') and scrambled control-sense (5'-UUCUCCGAACGUGUCACGU-3') were designed and synthesized by Shanghai GenePharma Co., Ltd. (Shanghai, China). Lipofectamine ${ }^{\circledR} 2000$ reagent (catalog no. 11668-019; Invitrogen; Thermo Fisher Scientific, Inc.) was used to perform cell transfection with siRNA or miRNA at a dose of $50 \mathrm{nM}$ and vectors at a dose of $15 \mathrm{nM}$. Cells transfected with scrambled negative control miRNA, scrambled control-sense or empty vector were used as negative control cells. Cells treated with Lipofectamine 2000 only were used as control cells. Overexpression rates of miRNA-10a and GLUT1 $>200 \%$ and a GLUT1-knockdown rate $<50 \%$ were reached $24 \mathrm{~h}$ after transfection, as revealed by RT-qPCR.

Cell proliferation assay. Cell proliferation was evaluated using Cell Counting Kit-8 (CCK-8; Beyotime Institute of Biotechnology, Haimen, China) $24 \mathrm{~h}$ after transfection. Briefly, cell suspensions with a cell density of $4 \times 10^{4}$ cells $/ \mathrm{ml}$ were prepared in Eagle's Minimum Essential Medium containing $2 \mathrm{mM} \mathrm{L}$-glutamine and 10\% FBS, and cells were transferred to a 96-well plate with $0.1 \mathrm{ml}$ cell suspension in each well. Cells were cultured at $37^{\circ} \mathrm{C}$ in a $5 \% \mathrm{CO}_{2}$ incubator, followed by the addition of $10 \mu \mathrm{lCCK}-8$ solution at $24,48,72$ and $96 \mathrm{~h}$. Subsequently, cells were cultured for a further $6 \mathrm{~h}$ and optical density values at $450 \mathrm{~nm}$ were measured using a Fisherbrand ${ }^{\mathrm{TM}}$ accuSkan $^{\mathrm{TM}}$ GO UV/Vis Microplate Spectrophotometer (Thermo Fisher Scientific, Inc.).

Glucose uptake assay. Glucose uptake abilities were measured by a glucose uptake assay $24 \mathrm{~h}$ after transfection. A total of $6 \times 10^{5}$ cells were harvested and washed twice with Krebs-Ringer-HEPES (KRH) buffer (25 mM Hepes, $\mathrm{pH} 7.4$, $120 \mathrm{mM} \mathrm{NaCl}, 1.2 \mathrm{mM} \mathrm{MgSO}_{4}, 5 \mathrm{mM} \mathrm{KCl} 1.3 \mathrm{mM} \mathrm{CaCl}_{2}$ and $\left.1.3 \mathrm{mM} \mathrm{KH}_{2} \mathrm{PO}_{4}\right)$ supplemented with $1 \mu \mathrm{Ci}[3 \mathrm{H}]$-2-deoxyglucose (PerkinElmer, Inc., Waltham, MA, USA). Glucose uptake was initiated by incubating cells at $37^{\circ} \mathrm{C}$ for $25 \mathrm{~min}$. Subsequently, cells were washed twice with ice-cold KRH buffer to stop glucose uptake. A liquid scintillation spectrometer was used to measure radioactivity and the $[3 \mathrm{H}]$-2-deoxyglucose content in cells was indicated by disintegrations per minute.

Total protein extraction and western blot analysis. The effect of miRNA-10a on GLUT1 expression was detected by western blot analysis. A Total Protein Extraction kit (catalog no. NBP2-37853; Novus Biologicals, Ltd., Cambridge, UK) was used to extract total protein from cells, according to the manufacturer's protocol. Electrophoresis was performed to separate denatured proteins using $10 \%$ SDS-PAGE gel with $20 \mu \mathrm{g}$ protein per lane. Following transfer to PVDF membranes, the membranes were blocked with 5\% non-fat milk for $2 \mathrm{~h}$ at room temperature. Western blot analysis was performed by incubation with rabbit anti-human GLUT1 (1:1,500; catalog no. ab15309; Abcam, Cambridge, UK) and rabbit anti-human GAPDH (1:1,300; catalog no. ab8245; Abcam) at $4^{\circ} \mathrm{C}$ overnight. The membranes were then incubated with goat anti-rabbit IgG-horseradish peroxidase secondary antibody (1:1,000; catalog no. MBS435036; MyBioSource, San Diego, CA, USA) at room temperature for $2 \mathrm{~h}$. An ECL ${ }^{\mathrm{TM}}$ Western Blotting Analysis system (Sigma-Aldrich; Merck $\mathrm{KGaA}$ ) was used to develop signals. Signals were normalized using Image J v1.46 software (National Institutes of Health, Bethesda, MD, USA). 

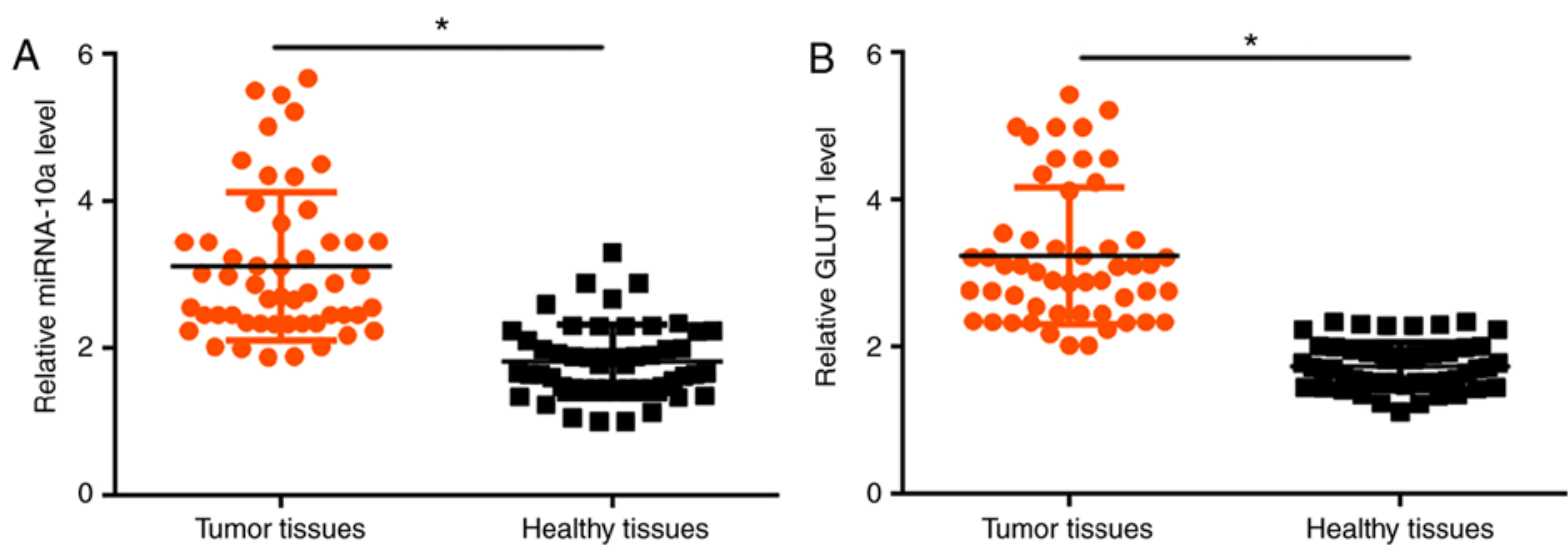

Figure 1. Expression levels of miRNA-10a and GLUT1 were upregulated in tumor tissues compared with adjacent healthy tissues. Expression levels of (A) miRNA-10a and (B) GLUT1 in tumor tissues and adjacent healthy tissues were measured by reverse transcription-quantitative polymerase chain reaction. Data are presented as the mean \pm standard deviation. ${ }^{*} \mathrm{P}<0.05$. miRNA-10a, microRNA-10a; GLUT1, glucose transporter 1.
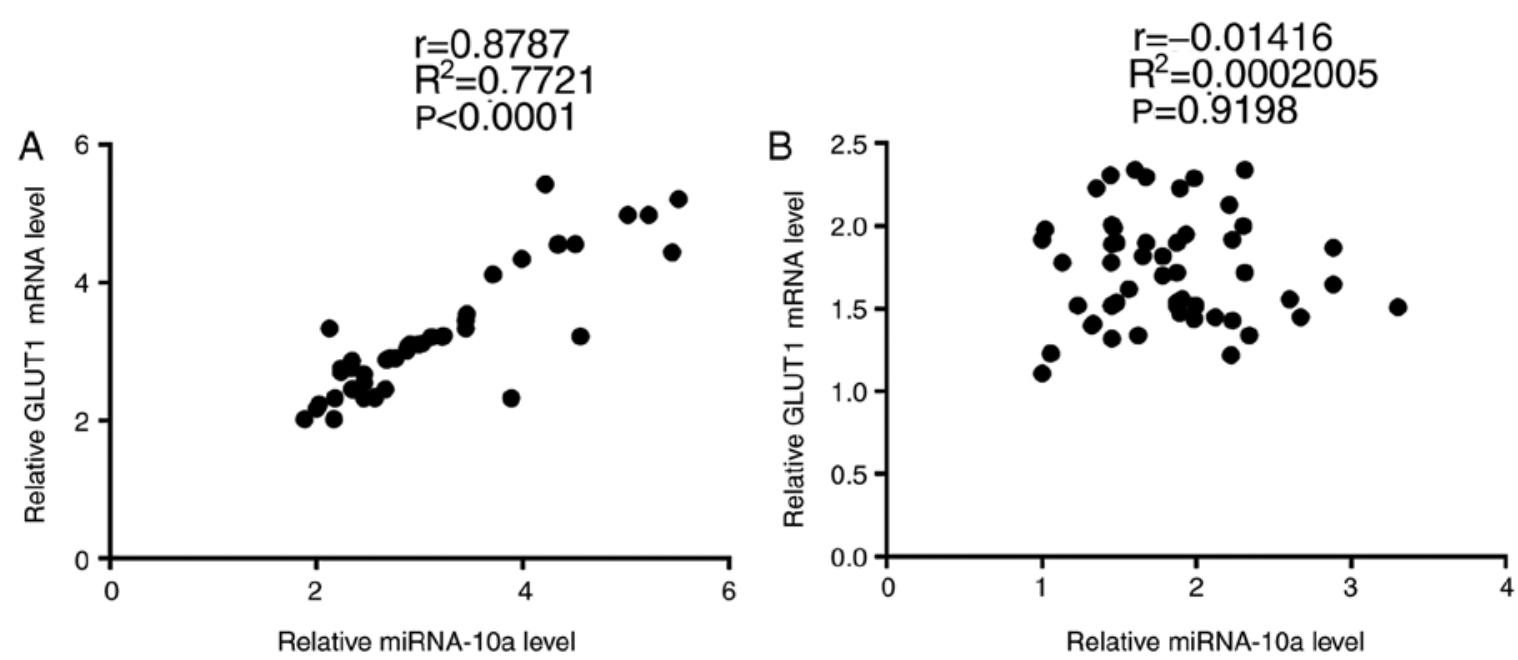

Figure 2. Expression levels of miRNA-10a and GLUT1 are positively correlated in tumor tissues but not in adjacent healthy tissues. Pearson's correlation coefficient analysis was performed to analyze the correlations between the expression levels of miRNA-10a and GLUT1 in (A) tumor tissues and (B) adjacent healthy tissues. miRNA-10a, microRNA-10a; GLUT1, glucose transporter 1.

Statistical analysis. All experiments were performed three times. Data are presented as the mean \pm standard deviation and were processed using GraphPad Prism 6 software (GraphPad Software, Inc., La Jolla, CA, USA). Correlation analysis between the expression levels of miRNA-10a and GLUT1 was performed using Pearson's correlation coefficient. Comparisons of the expression levels of miRNA-10a and GLUT1 between tumor tissues and adjacent healthy tissues were performed using a paired Student's t-test. Comparisons among multiple groups were performed by one-way analysis of variance followed by Tukey's test. $\mathrm{P}<0.05$ was considered to indicate a statistically significant difference.

\section{Results}

Expression levels of miRNA-10a and GLUT1 are upregulated in tumor tissues compared with adjacent healthy tissues. The expression levels of miRNA-10a and GLUT1 in tumor tissues and adjacent healthy tissues obtained from patients with OSCC were detected by RT-qPCR. Compared with adjacent healthy tissues, the expression level of miRNA-10a was significantly increased in tumor tissues $(\mathrm{P}<0.05$; Fig. 1A). In addition, the expression of GLUT1 was significantly upregulated in tumor tissues compared with adjacent healthy tissues $(\mathrm{P}<0.05$; Fig. 1B).

Expression levels of miRNA-10a and GLUT1 are positively correlated in tumor tissues but not in adjacent healthy tissues. Correlation analyses between the expression levels of miRNA-10a and GLUT1 were performed using Pearson's correlation coefficient. A significant positive correlation was revealed between the expression levels of miRNA-10a and GLUT1 in tumor tissues $(\mathrm{P}<0.001$; Fig. $2 \mathrm{~A})$. By contrast, a significant correlation was not identified between the expression levels of miRNA-10a and GLUT1 in adjacent healthy tissues ( $\mathrm{P}=0.9198$; Fig. 2B).

miRNA-10a overexpression upregulates GLUT1 in the OSCC SCCO90 and SCC25 cell lines. To further investigate the association between miRNA-10a and GLUT1, miRNA-10a mimic and a GLUT1 expressing vector were transfected into SCC090 and SCC25 OSCC cell lines, followed by the 
A

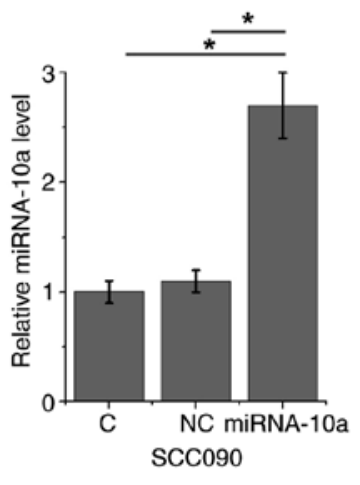

B

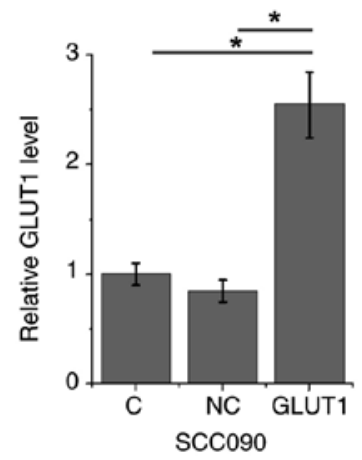

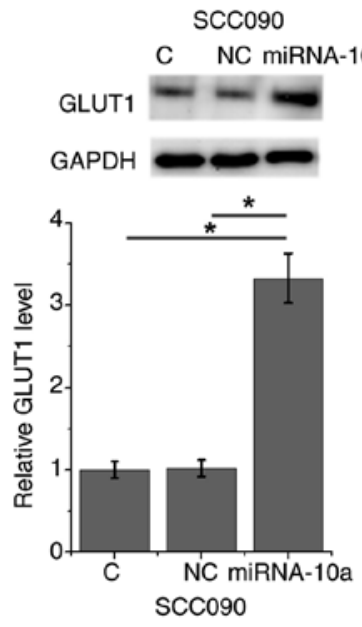
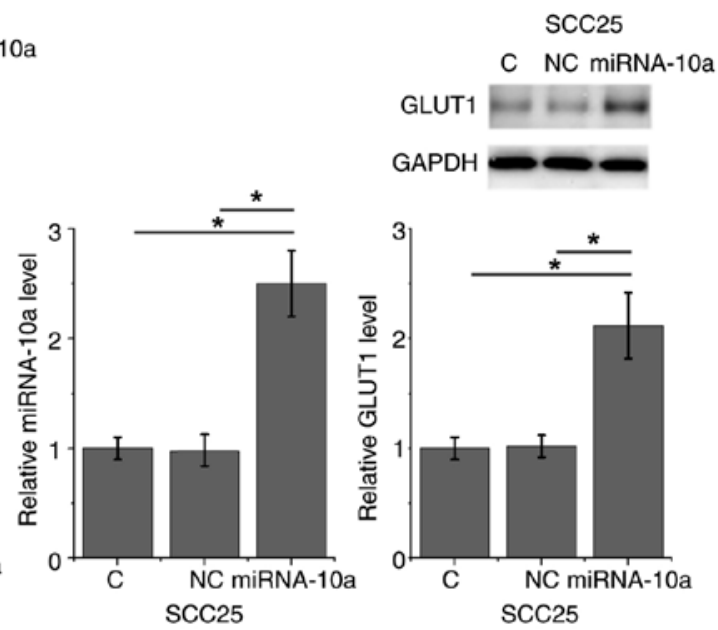

$\operatorname{SCC} 25$
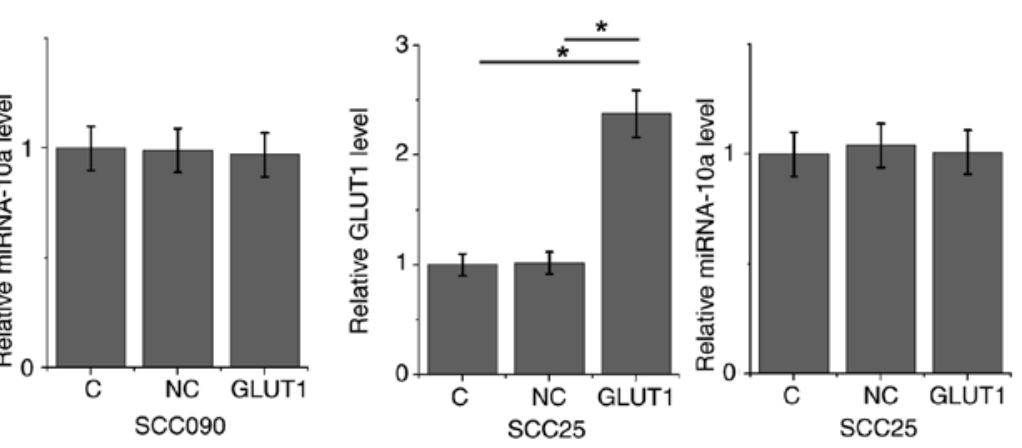

Figure 3. miRNA-10a overexpression upregulates GLUT1 in SCC090 and SCC25 OSCC cell lines. miRNA-10a and GLUT1 overexpression were achieved in SCC090 and SCC25 cells. (A) The effects of miRNA-10a overexpression on GLUT1 expression were detected by western blot analysis. (B) The effects of GLUT1 overexpression on the expression of miRNA-10a were detected by reverse transcription-quantitative polymerase chain reaction. Data are presented as the mean \pm standard deviation. ${ }^{*} \mathrm{P}<0.05$. C, control; NC, negative control; miRNA-10a, microRNA-10a; GLUT1, glucose transporter 1.

detection of GLUT1 protein and miRNA-10a expression levels by RT-qPCR and western blot, respectively. Compared with the control and negative control cells, miRNA-10a and GLUT1 were significantly increased at $24 \mathrm{~h}$ after transfections (Fig. 3), indicating the transfections were successful. miRNA-10a overexpression significantly upregulated the expression of GLUT1 in SCC090 and SCC25 cells $(\mathrm{P}<0.05$; Fig. 3A). By contrast, GLUT1 overexpression did not significantly affect the expression of miRNA-10a in SCC090 and SCC25 cells (Fig. 3B).

miRNA-10a overexpression promotes glucose uptake and cell proliferation via GLUT1. The expression levels of GLUT1 in SCC090 and SCC25 cells transfected with GLUT1 siRNA were significantly decreased compared with the control and negative control cells (Fig. 4). Compared with the control and negative control cells, overexpression of miRNA-10a and GLUT1 significantly promoted glucose uptake, while GLUT1-knockdown significantly inhibited glucose uptake in SCC090 and SCC25 cells ( $\mathrm{P}<0.051$ Fig. 5A). In addition, GLUT1-knockdown significantly attenuated the enhancing effects of miRNA-10a overexpression on glucose uptake $(\mathrm{P}<0.05)$. Furthermore, GLUT1 overexpression significantly promoted the proliferation of SCC090 and SCC25 cells, while
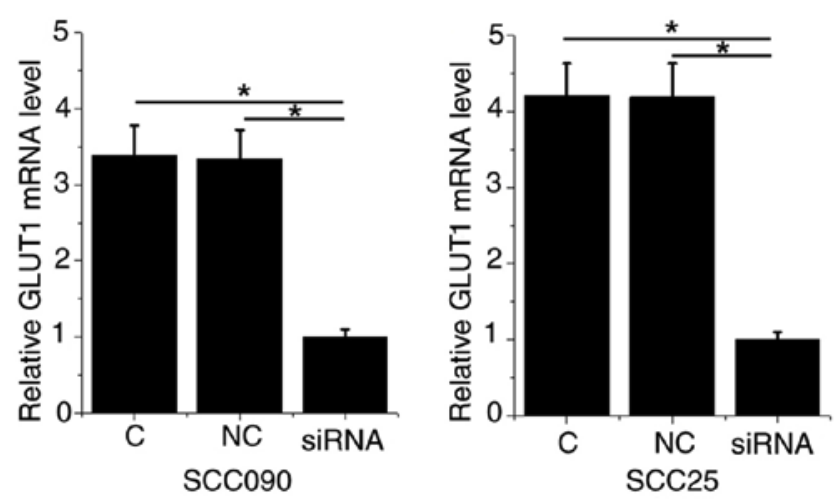

Figure 4. Knockdown of GLUT1 in SCC090 and SCC25 OSCC cell lines. Transfection with GLUT1 siRNA significantly decreased the expression level of GLUT1 in SCC090 and SCC25 cells compared with the controls. Data are presented as the mean \pm standard deviation. ${ }^{*} \mathrm{P}<0.05$. C , control; $\mathrm{NC}$, negative control; GLUT1, glucose transporter 1; siRNA, small interfering RNA.

GLUT1-knockdown significantly inhibited the proliferation of SCC090 and SCC25 cells ( $\mathrm{P}<0.05$; Fig. 5B). Additionally, GLUT1-knockdown significantly attenuated the enhancing effects of miRNA-10a overexpression on cell proliferation $(\mathrm{P}<0.05)$. 

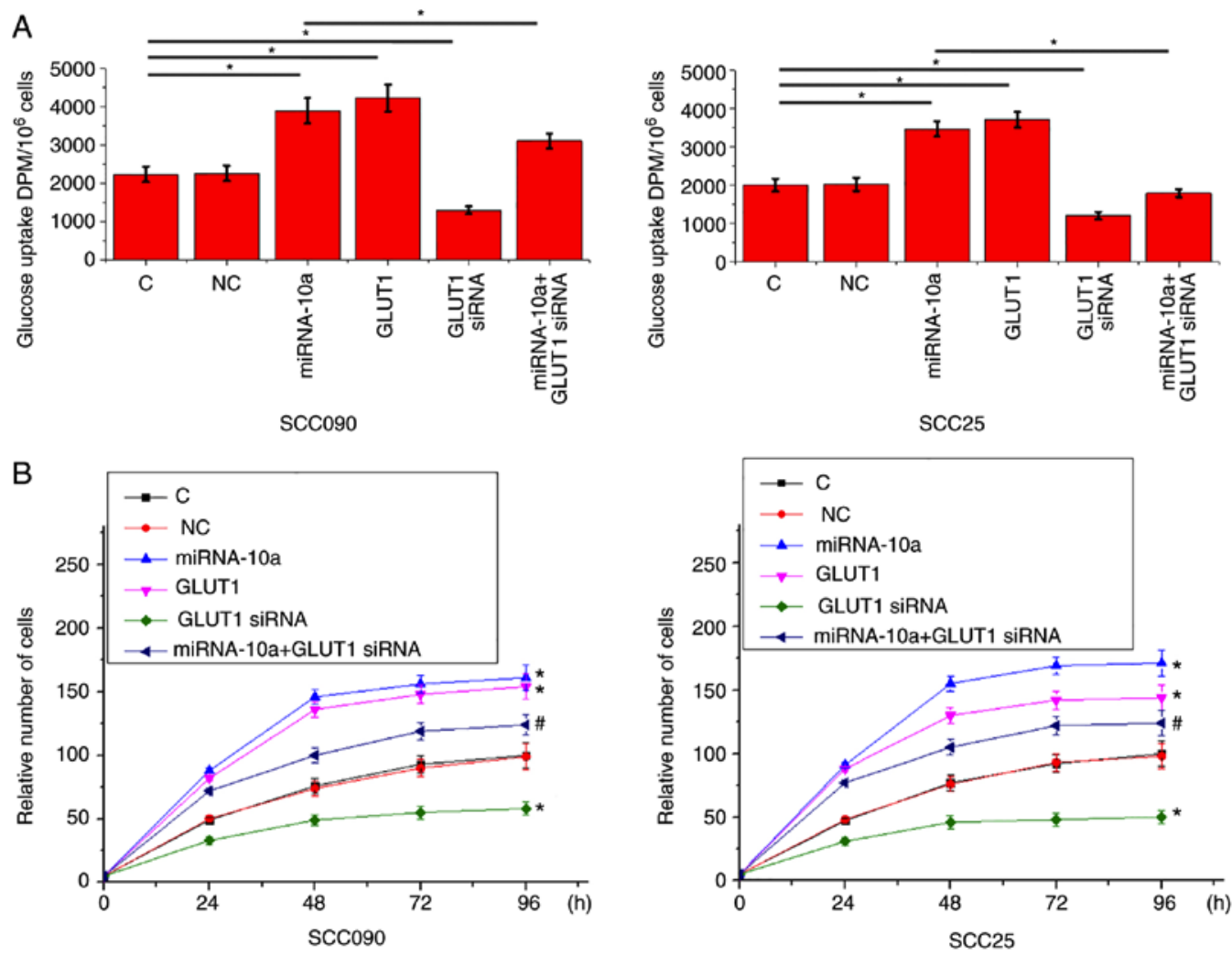

Figure 5. miRNA-10a overexpression promotes glucose uptake and cell proliferation via GLUT1. miRNA-10a and GLUT1 overexpression were achieved in SCC090 and SCC25 cells. (A) The effects of miRNA-10a and GLUT1 overexpression on glucose uptake in SCC090 and SCC25 cells were detected by a glucose uptake assay. ${ }^{*} \mathrm{P}<0.05$. (B) The effects of miRNA-10a and GLUT1 overexpression, and GLUT1-knockdown on the proliferation of SCC090 and SCC25 cells were detected by Cell Counting Kit-8 assay. Data are presented as the mean \pm standard deviation. ${ }^{*} \mathrm{P}<0.05$ vs. C; ${ }^{\#} \mathrm{P}<0.05$ vs. miRNA-10a overexpression group. C, control; NC, negative control; miRNA-10a, microRNA-10a; GLUT1, glucose transporter 1; siRNA, small interfering RNA; DPM, disintegrations per minute.

\section{Discussion}

A previous study characterized miRNA-10a as an oncogenic miRNA in lung cancer (14); however, to the best of our knowledge, the role of miRNA-10a in other human diseases remains unknown. The present study demonstrated that miRNA-10a is also likely an oncogenic miRNA in OSCC, which is a major type of oral cancer that accounted for $<95 \%$ of oral cancer cases worldwide in recent years $(4,5)$. The role of miRNA-10a in the proliferation of OSCC cells is likely achieved via an upregulation of GLUT1 and accelerated glucose uptake.

An upregulation of GLUT1 is frequently observed during the development of different types of human cancer, including OSCC (16). Overexpression of GLUT1 promotes glucose metabolism, which provides energy for cancer cell division and proliferation (17). Therefore, inhibition of GLUT1 is considered as a potential therapeutic target for the treatment of different types of cancer (18). Upregulation of GLUT1 also promotes the growth of oral tumors (19). Consistent with previous studies, the present study demonstrated that the expression level of GLUT1 was significantly higher in tumor tissues compared with adjacent healthy tissues of patients with OSCC. In vitro cell experiments also revealed that overexpression of GLUT1 promoted cancer cell proliferation and glucose uptake, while silencing of GLUT1 with siRNA inhibited cancer cell proliferation and glucose uptake. These data further suggested an oncogenic role of GLUT1 in oral cancer.
miRNAs serve key roles in the regulation of glucose metabolism in cancer cells $(20,21)$. As an oncogenic miRNA, miRNA-10a is upregulated in lung cancer (14); however, to the best of our knowledge, its expression pattern in OSCC remains unknown. The present study demonstrated that miRNA-10a was upregulated in tumor tissues compared with adjacent healthy tissues of patients with OSCC. In addition, in vitro cell experiments revealed that miRNA-10a promoted cancer cell proliferation and glucose uptake. Therefore, miRNA-10a may serve an oncogenic role in OSCC by upregulating glucose uptake and accelerating cell proliferation.

The expression of GLUT1 is regulated by miRNAs during cancer progression (13). The present study identified a significant positive correlation between the expression levels of miRNA-10a and GLUT1 in tumor tissues. The in vitro experiments revealed that overexpression of miRNA-10a could significantly mediate the upregulation of GLUT1, while overexpression of GLUT1 did not significantly alter the expression of miRNA-10a. Furthermore, silencing of GLUT1 with siRNA significantly attenuated the enhancing effects of miRNA-10a overexpression on cancer cell proliferation and glucose uptake. Therefore, miRNA-10a may promote cancer cell proliferation and glucose uptake in OSCC by serving as an upstream activator of GLUT1. Previous studies have also reported that miRNAs participate in the growth, development and progression of cancer by regulating the expression of GLUT1 $(13,22)$. In summary, the current study identified a novel miRNA regulator of GLUT1 in cancer biology. 
The present study is limited by the small sample size. Therefore, studies with a larger sample size and further correlation analyses are required. Notably, there is no promising binding site of miRNA-10a in GLUT1 based on a local blast analysis. In addition, the correlation between miRNA-10a and GLUT1 expression levels was not significant in adjacent normal tissues. Therefore, miRNA-10a may be not able to directly regulate GLUT1 and the interaction between miRNA-10a and GLUT1 may be mediated by certain pathological factors, such as tumor suppressive or oncogenic pathways. The present study failed to perform miR-10a silencing experiments due to a low knockdown efficiency; therefore, this should be improved in future studies.

In conclusion, miRNA-10a was identified to be upregulated in OSCC. miRNA-10a may promote cancer cell proliferation and glucose uptake in OSCC by acting as an upstream activator of GLUT1.

\section{Acknowledgements}

Not applicable.

\section{Funding}

No funding was received.

\section{Availability of data and materials}

The analyzed datasets generated during the present study are available from the corresponding author on reasonable request.

\section{Authors' contributions}

YC and WC designed experiments. YC and YS performed experiments. YY and XT analyzed data. WC drafted the manuscript and all authors approved this manuscript.

\section{Ethics approval and consent to participate}

The present study was approved by the Ethics Committee of Nanjing Stomatological Hospital (Nanjing, China). All patients provided written informed consent prior to their inclusion in the study.

\section{Patient consent for publication}

Not applicable.

\section{Competing interests}

The authors declare that they have no competing interests.

\section{References}

1. Manikandan M, Deva Magendhra Rao AK, Arunkumar G, Manickavasagam M, Rajkumar KS, Rajaraman R and Munirajan AK: Oral squamous cell carcinoma: microRNA expression profiling and integrative analyses for elucidation of tumourigenesis mechanism. Mol Cancer 15: 28, 2016.

2. Weatherspoon DJ, Chattopadhyay A, Boroumand S and Garcia I: Oral cavity and oropharyngeal cancer incidence trends and disparities in the United States: 2000-2010. Cancer Epidemiol 39: 497-504, 2015.
3. Tota JE, Anderson WF, Coffey C, Califano J, Cozen W, Ferris RL, St John M, Cohen EE and Chaturvedi AK: Rising incidence of oral tongue cancer among white men and women in the United States, 1973-2012. Oral Oncol 67: 146-152, 2017.

4. Alnuaimi AD, Wiesenfeld D, O'Brien-Simpson NM, Reynolds EC and McCullough MJ: Oral Candida colonization in oral cancer patients and its relationship with traditional risk factors of oral cancer: A matched case-control study. Oral Oncol 51: 139-145, 2015.

5. Williams HK: Molecular pathogenesis of oral squamous carcinoma. Mol Pathol 53: 165-172, 2000.

6. Zheng J: Energy metabolism of cancer: Glycolysis versus oxidative phosphorylation. Oncol Lett 4: 1151-1157, 2012.

7. Rodríguez-Enríquez S, Marín-Hernández A, Gallardo-Pérez JC, Carreño-Fuentes L and Moreno-Sánchez R: Targeting of cancer energy metabolism. Mol Nutr Food Res 53: 29-48, 2009.

8. Olson AL and Pessin JE: Structure, function, and regulation of the mammalian facilitative glucose transporter gene family: Annu Rev Nutr 16: 235-256, 1996.

9. Krzeslak A, Wojcik-Krowiranda K, Forma E, Jozwiak P, Romanowicz H, Bienkiewicz A and Brys M: Expression of GLUT1 and GLUT3 glucose transporters in endometrial and breast cancers. Pathol Oncol Res 18: 721-728, 2012.

10. Carvalho KC, Cunha IW, Rocha RM, Ayala FR, Cajaíba MM, Begnami MD, Vilela RS, Paiva GR, Andrade RG and Soares FA: GLUT1 expression in malignant tumors and its use as an immunodiagnostic marker. Clinics 66: 965-972, 2011.

11. Hayes J, Peruzzi PP and Lawler S: MicroRNAs in cancer: Biomarkers, functions and therapy. Trends Mol Med 20: 460-469, 2014.

12. Chen B, Li H, Zeng X, Yang P, Liu X, Zhao X and Liang S: Roles of microRNA on cancer cell metabolism. J Transl Med 10: 228, 2012.

13. Liu M, Gao J, Huang Q, Jin Y and Wei Z: Downregulating microRNA-144 mediates a metabolic shift in lung cancer cells by regulating GLUT1 expression. Oncol Lett 11: 3772-3776, 2016.

14. Yu T, Liu L, Li J, Yan M, Lin H, Liu Y, Chu D, Tu H, Gu A and Yao M: MiRNA-10a is upregulated in NSCLC and may promote cancer by targeting PTEN. Oncotarget 6: 30239-30250, 2015.

15. Livak KJ and Schmittgen TD: Analysis of relative gene expression data using real-time quantitative PCR and the 2- $\Delta \Delta \mathrm{CT}$ method. Methods 25: 402-408, 2001.

16. Wang J, Ye C, Chen C, Xiong H, Xie B, Zhou J, Chen Y, Zheng S and Wang L: Glucose transporter GLUT1 expression and clinical outcome in solid tumors: A systematic review and meta-analysis. Oncotarget 8: 16875-16886, 2017.

17. Oh S, Kim H, Nam KS and Shin I: Glut1 promotes cell proliferation, migration and invasion by regulating epidermal growth factor receptor and integrin signaling in triple-negative breast cancer cells. BMB Rep 50: 132-137, 2017.

18. Shibuya K, Okada M, Suzuki S, Seino M, Seino S, Takeda H and Kitanaka C: Targeting the facilitative glucose transporter GLUT1 inhibits the self-renewal and tumor-initiating capacity of cancer stem cells. Oncotarget 6: 651-661, 2015.

19. Kraus D, Reckenbeil J, Wenghoefer M, Stark H, Frentzen M, Allam JP, Novak N, Frede S, Götz W, Probstmeier R, et al: Ghrelin promotes oral tumor cell proliferation by modifying GLUT1 expression. Cell Mol Life Sci 73: 1287-1299, 2016.

20. Chen B, Liu Y, Jin X, Lu W, Liu J, Xia Z, Yuan Q, Zhao X, $\mathrm{Xu} \mathrm{N}$ and Liang S: MicroRNA-26a regulates glucose metabolism by direct targeting PDHX in colorectal cancer cells. BMC Cancer 14: 443, 2014.

21. Lv X, Yao L, Zhang J, Han P and Li C: Inhibition of microRNA-155 sensitizes lung cancer cells to irradiation via suppression of HK2-modulated glucose metabolism. Mol Med Rep 14: 1332-1338, 2016.

22. Li P, Yang X, Cheng Y, Zhang X, Yang C, Deng X, Li P, Tao J, Yang $\mathrm{H}$, Wei J, et al: MicroRNA-218 increases the sensitivity of bladder cancer to cisplatin by targeting Glut. Cell Physiol Biochem 41: 921-932, 2017.

This work is licensed under a Creative Commons Attribution-NonCommercial-NoDerivatives 4.0 International (CC BY-NC-ND 4.0) License. 